Check for updates

Cite this: Soft Matter, 2018, 14,5525

Received 11th April 2018, Accepted 7th June 2018

DOI: $10.1039 / c 8 s m 00755 a$

rsc.li/soft-matter-journal

\title{
Self-assembly and surface behaviour of pure and mixed zwitterionic amphiphiles in a deep eutectic solvent $\dagger$
}

\author{
A. Sanchez-Fernandez, (D) $\ddagger^{a b}$ G. L. Moody, $\ddagger^{a}$ L. C. Murfin, (D) $\ddagger^{a}$ T. Arnold, (D) abc \\ A. J. Jackson, (D) *bd S. M. King, (D) ${ }^{\text {e }}$ S. E. Lewis (D) and K. J. Edler (D) ${ }^{a}$
}

\begin{abstract}
Recent investigations have shown that deep eutectic solvents provide a suitable environment for selforganisation of biomolecules, in particular phospholipids and proteins. However, the solvation of complex lyophilic moieties by deep eutectic solvents still remains unclear. Here we explore the behaviour of zwitterionic surfactants in choline chloride:glycerol eutectic mixture. Dodecyl-2-(trimethylammonio)ethylphosphate and $\mathrm{N}$-alkyl-N,N-dimethyl-3-ammonio-1-propanesulfonate (alkyl = dodecyl, tetradecyl) surfactants were investigated by means of surface tension, $\mathrm{X}$-ray reflectivity and small-angle neutron scattering. These surfactants were found to remain surface active and form globular micelles in deep eutectic solvents. Still, the surface behaviour of these species was found to differ depending on the headgroup and tail structure. The morphology of the micelles also slightly varies between surfactants, demonstrating differences in the packing of individual monomers. The characteristics of mixtures of the dodecyl surfactants is also reported, showing a deviation from ideal mixing associated with attractive interactions between sulfobetaine and phosphocholine headgroups. Such non-ideality results in variation of the surface behaviour and self-assembly of these surfactant mixtures. The results presented here will potentially lead to the development of new alternatives for drug-delivery, protein solubilisation and biosensing through a better fundamental understanding of the behaviour of zwitterionic surfactants in deep eutectic solvents.
\end{abstract}

\section{Introduction}

Deep eutectic solvents (DES) have emerged as an alternative to traditional solvents in many applications. ${ }^{1,2}$ DES are green solvents obtained through the complexation of naturally occurring salts with compounds, such as sugar, alcohols, amines and carboxylic acids, among others. ${ }^{1,3-5}$ Furthermore through different combinations of precursors the properties of the solvent can be tailored, ${ }^{1}$ potentially providing sustainable solvents tailored for particular applications. Understanding the microscopic

\footnotetext{
${ }^{a}$ Department of Chemistry, University of Bath, Claverton Down, Bath, BA2 7AY, UK

${ }^{b}$ European Spallation Source, SE-221 00, Lund, Sweden.

E-mail: andrew.jackson@esss.se

${ }^{c}$ Diamond Light Source, Harwell Campus, Didcot OX11 ODE, UK

${ }^{d}$ Department of Physical Chemistry, Lund University, SE-221 00, Lund, Sweden

${ }^{e}$ ISIS Pulsed Neutron \& Muon Source, Harwell Campus, Didcot OX11 OQX, UK

$\dagger$ Electronic supplementary information (ESI) available: Surfactant synthesis and characterization, X-ray reflectivity model test, SANS model test and plots from isotopic mixtures not shown in the main text. Data supporting this article has been made freely available via the University of Bath Research Data Archive system at DOI: $10.15125 /$ BATH-00523. See DOI: $10.1039 / \mathrm{c} 8 \mathrm{sm} 00755 \mathrm{a}$

‡ A. S.-F., G. L. M. and L. M. have equally contributed to the work presented here.
}

structure of the solvent and dynamics represents an essential step to predict and understand the macroscopic behaviour of the solvent, and this is the subject of considerable recent effort. ${ }^{6-9}$ Such investigations have shown that an extensive hydrogen bond interaction between the DES precursors is responsible for the formation and stability of the solvent. Thus, DES provides a H-bonding environment analogous to that in water.

These solvents have been suggested as a non-aqueous environment where biomolecules may retain partial or total activity, ${ }^{2,10,11}$ presumably due to the existence of such hydrogen bond networks in DES. The investigation of biomolecules and bioprocesses involving DES has recently experienced a major upsurge, with relevant studies published on vesicle formation, phospholipid bilayers, DNA structuring and protein conformation and activity. ${ }^{12-20}$

Zwitterionic surfactants are molecules that contain both positively and negatively charged chemical groups within their headgroup structure. Such moieties are ubiquitous in biological systems, from proteins to phospholipid membranes. Naturally occurring di-chain phospholipids are the main components of cell membranes. As such, these are often used in simplified model systems such as phospholipid monolayers or bilayers, 
to investigate the properties of highly complex biological membranes. $^{21,22}$ It is also possible to synthesise zwitterionic surfactants with single chain architectures and such molecules may open up new possibilities for applications in drug delivery, biosensors, protein stabilisation or those that require high degrees of biocompatibility. ${ }^{23-25}$

In this work we have investigated two classes of single chain zwitterionic surfactants; phosphocholine and sulfobetaine surfactants. The behaviour of these molecules is relatively well understood in water and as such they are good model systems to understand the effects of the DES solvent. Zwitterionic surfactants generally show high solubility in water, broad isoelectric ranges, and high resistance to changes in the $\mathrm{pH}$ and ionic strength of the media, ${ }^{26,27}$ and the surfactants studied here have been shown to form micelles in water. ${ }^{27-30}$

DES have been recently demonstrated to support surfactant self-assembly of cationic and anionic surfactants, ${ }^{31-33}$ as well as the formation of thermodynamically stable phospholipid vesicles. ${ }^{12,19}$ Such investigations provide new alternatives for applications in surfactant templating of nanostructured materials, formulations and drug delivery. However, the solvation of surfactants in DES is not yet fully understood. Our hope is that this study will add to this growing body of evidence to open new prospects in tailorable self-assembled systems. Here we examine three different zwitterionic surfactants in 1:2 choline chloride: glycerol. ${ }^{34}$ Choline chloride:glycerol DES was selected as the solvent for this investigation as this has been previously reported as a suitable environment for both anionic and cationic moieties. ${ }^{33,35}$ These surfactants are dodecyl-2-(trimethylammonio)ethylphosphate ( $\mathrm{C}_{12}$-PC), $\mathrm{N}$-dodecyl- $N, N$-dimethyl-3-ammonio-1-propanesulfonate (SB3-12) and $N$-tetradecyl- $N, N$-dimethyl-3-ammonio-1-propanesulfonate (SB3-14). The structure of these surfactants is presented in Fig. 1. Note that these two headgroup types have the opposite orientation of charge separation relative to the alkyl tail. They have been studied by means of surface tension, X-ray reflectivity (XRR) and small-angle neutron scattering (SANS). These experiments have been performed at a temperature above room temperature $\left(50{ }^{\circ} \mathrm{C}\right)$ to avoid the crystallisation of the $\mathrm{C} 14$ surfactant below its Krafft temperature. Furthermore, mixtures of $\mathrm{C}_{12}-\mathrm{PC}$ and SB3-12 surfactants were studied at different molar ratios in order to explore whether the relative interactions between the ionic headgroups synergistically influence aggregate behaviour. ${ }^{36}$

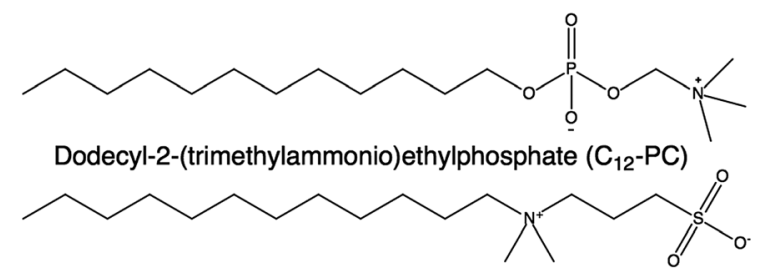

$N$-dodecyl- $N, N$-dimethyl-3-ammonio-1-propanesulfonate (SB3-12)

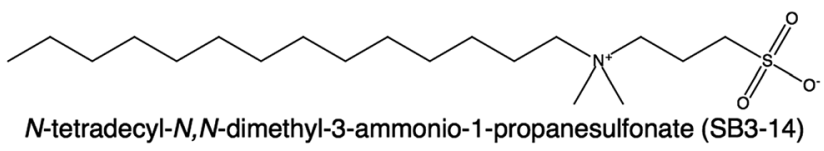

Fig. 1 Molecular structures of $\mathrm{C}_{12}-\mathrm{PC}, \mathrm{SB} 3-12$ and SB3-14 surfactants.

\section{Experimental}

\section{Materials}

Choline chloride (>98\%, Sigma, h-ChCl) and glycerol ( $>99 \%$, Sigma, h-Glyc) were mixed in a $1: 2$ molar ratio at $80{ }^{\circ} \mathrm{C}$ on a hotplate until a transparent, homogeneous liquid was obtained. The deuterated version of the DES was prepared following the same procedure as above with $\mathrm{d}_{9}$-choline chloride (99\%, 99.9\%D, d-ChCl, Cambridge Isotope Laboratory) and $\mathrm{d}_{8}$-glycerol (98\%, 99\%D, d-Glyc, Cambridge Isotope Laboratory). Solvents were equilibrated at $40{ }^{\circ} \mathrm{C}$ for 24 hours. In an attempt to control and minimise the water content in the systems, solvents were freeze-dried prior to surfactant solution preparation, sealed and stored under a dry atmosphere. The purity of the solvents was checked through NMR and Karl-Fischer titration (Mettler Toledo DL32 Karl-Fischer Coulometer Aquiline electrolyte A (Fisher Scientific), Aqualine Catholyte CG A). Measurements on aliquots of pure solvent stored under the same conditions as the samples were characterized and showed that the water content of the system was maintained below $\sim 3000 \mathrm{ppm}(0.3 \mathrm{wt} \%)$ during the experimental procedures presented here.

The sulfobetaine surfactants (SB3-12 and SB3-14) were synthesised following the procedure from Qu et al. ${ }^{28}$ After synthesis the surfactants were purified via recrystallization with hot methanol/acetone. The purity of the final products was assessed by ${ }^{1} \mathrm{H}$ NMR, ${ }^{13} \mathrm{C}$ NMR and High Resolution Mass Spectrometry. A detailed description of the synthesis procedure and the results from the characterisation of the final products are included in the ESI. $\dagger$ Dodecyl-2-(trimethylammonio)ethylphosphate (>99\%) was supplied by Glycon Biochemicals GmbH and used without further purification.

\section{Methods}

Surface tension. Surface tension was determined using the drop shape analysis method. Surface tension measurements were carried on a Krüss Drop-Shape Analyser (DSA-100) at Diamond Light Source, UK. The samples were equilibrated in the oven at $60{ }^{\circ} \mathrm{C}$ prior to measurement. Drops of different surfactant concentrations were suspended using a needle and allowed to equilibrate. Pictures of various drops were taken and the contours of these were fitted using the Young-Laplace equation. The interfacial tension between air and the solution was calculated from those fits. Each concentration of surfactant was measured at least five times and the final value of surface tension was obtained as the average of those values. The temperature could not be controlled, however, although this may have an effect upon the measurements taken, our results were shown to be self-consistent throughout all of the measurements.

Samples for surface tension measurements were prepared by dilution of high concentration stock solutions. These stock solutions were prepared by direct mixing each surfactant with DES and subsequently diluted using pure solvent in order to obtain lower concentrations, minimising the variability between samples. Surfactant mixtures were prepared at different surfactant molar ratios following the same procedure, $\mathrm{C}_{12}-\mathrm{PC} / \mathrm{SB} 3-12$ : $0.2 / 0.8,0.35 / 0.65,0.5 / 0.5,0.65 / 0.35$ and $0.8 / 0.2$. 
X-ray reflectivity. The behaviour of the surfactants at the airDES interface was characterised by means of X-ray reflectivity (XRR). Experimental data were taken on I07 beamline at the Diamond Light Source, UK. ${ }^{37}$ The experiment was performed at a $12.5 \mathrm{keV}$ photon energy using the double-crystal-deflector system to deflect the incoming beam and enable the investigation of interfaces by varying the incoming angle. ${ }^{37}$ The reflected intensity was measured using a Pilatus 100k detector with one "region-of-interest" (ROI) for the reflected intensity and another ROI of the same size displaced vertically for the background. Scattering data were collected over 4 different attenuation regimes to cover the wide dynamic range of a reflectivity curve over a momentum transfer $(q)$ range of 0.01 to $0.72 \AA^{-1}$. Data were reduced and normalised accounting for the incident flux, attenuation factors, a footprint over-illumination correction and a background subtraction using the standard procedures of the beamline. ${ }^{37}$ Samples were placed in a temperature-controlled PTFE trough, volume $30 \mathrm{ml}$, under a dry helium atmosphere and the temperature was maintained at $50{ }^{\circ} \mathrm{C}$ during sample equilibration (at least 1 hour) and data collection.

Samples for reflectivity measurements of pure surfactants and surfactant mixtures in DES were prepared at the critical micelle concentration (CMC) of each system. Pure hydrogenous surfactant powders or homogenous mixtures of surfactants were mixed with hydrogenous solvent and subsequently equilibrated for 24 hours at $50{ }^{\circ} \mathrm{C}$ before measurement.

XRR data were fitted using the Abelés formalism implemented in Motofit. ${ }^{38,39}$ This method, also known as the Dynamic Approximation, uses classical optics to simulate the reflectivity pattern from a given electron density profile. A two-layer plus subphase geometry was found as the simplest model that allowed fitting of the data from pure phosphocholine surfactant solutions, whereas one layer was enough to satisfactorily fit data from pure sulfobetaine solutions. Whilst the use of a onelayer model for the sulfobetaine systems in water has been previously validated, ${ }^{40}$ here, data have been fitted using the two-layer plus subphase model in order to make the results from all of the systems presented directly comparable. The model uses a layer to describe the "air-solvated" tail-region of the surfactant and a headgroup layer containing a certain amount of DES. An infinitely thick subphase is used to describe the solvent. The parameters used to describe the layers are: thickness $(t)$, scattering length density of the layer (SLD) and roughness $(\sigma)$, where the subscripts $s, t$ and $h$ stand for solvent, tails and headgroup layers respectively. The SLDs of the headgroup and tail of each surfactant were calculated by accounting for the scattering length of each group and the volume it occupies (see ESI $\dagger$ for further details). The SLDs used in the model therefore account for the amount of surfactant in each layer, allowing calculation of the volume fraction of each component in the layer: volume fraction of tails $\left(\phi_{\mathrm{t}}\right)$ in the tail layer, and volume fraction of headgroups $\left(\phi_{\mathrm{h}}\right)$ and solvent $\left(\phi_{\mathrm{s}}\right)$ in the headgroup layer. The subphase is described by the SLD $\left(\mathrm{SLD}_{\mathrm{s}}\right)$ and the roughness of the solvent $\left(\sigma_{\mathrm{s}}\right)$. A background term is used to account for the residual background that remains after data subtraction.
To be physically realistic, the model is constrained to ensure that surface excess of both layers is the same, i.e. the two layers contain the same number of headgroups and tails. ${ }^{41}$ This has been done through the introduction of the following mathematical constraint.

$$
1-\phi_{\mathrm{s}}=\frac{\mathrm{SLD}_{\mathrm{t}} t_{\mathrm{t}} b_{\mathrm{h}}}{\mathrm{SLD}_{\mathrm{h}} t_{\mathrm{h}} b_{\mathrm{t}}}
$$

where $b$ refers to the scattering length of either the tails or the head, and $\phi_{\mathrm{s}}$ is the fractional solvent volume in the head layer. The surfactant area per molecule (APM, $\AA^{2}$ ) and surface excess concentration $\left(\Gamma_{\mathrm{S}}\right)$ were subsequently calculated using the following equations:

$$
\begin{aligned}
& \mathrm{APM}=\frac{b_{\mathrm{t}}}{\mathrm{SLD}_{\mathrm{t}} t_{\mathrm{t}}} \\
& \Gamma_{\mathrm{S}}=\frac{10^{20}}{\operatorname{APM} N_{\mathrm{A}}}
\end{aligned}
$$

where $N_{\mathrm{A}}$ is Avogadro's number.

Small-angle neutron scattering. SANS measurements were performed on the LOQ diffractometer at the ISIS Pulsed Neutron and Muon Facility, UK. ${ }^{42}$ LOQ is a time-of-flight instrument with fixed sample-to-detector distances of $0.5 \mathrm{~m}$ and $4 \mathrm{~m}$. A "white" neutron beam of wavelength $2.2 \leq \lambda \leq 10.0$ A was used during the experiment, providing a simultaneous $q$-range of 0.007-1 $\AA^{-1}$. Samples were loaded in $1 \mathrm{~mm}$ path length, $1 \mathrm{~cm}$ width, quartz cells (Hellma $\mathrm{GmbH})$ and placed in an automated temperature controlled sample changer for measurement. The temperature was kept at $50{ }^{\circ} \mathrm{C}$ throughout the measurements, in order to maintain samples equilibrated and above the Kraft temperature of the surfactants.

The data collected were reduced to absolute units $\left(I(q), \mathrm{cm}^{-1}\right.$ vs. $q, \AA^{-1}$ ) following the standard procedures on the instrument using the Mantid framework. ${ }^{43,44}$ Data were corrected for detector efficiency, background noise, sample transmission and the scattering from an empty cell, after which the intensity was place on an absolute scale by reference to the scattering from a partially-deuterated polystyrene blend of known molecular weight. The contribution from the solvent was then subtracted from each sample accounting for the incoherent contribution using SasView 4.1, using a procedure previously described. ${ }^{45}$

Samples of the pure surfactants for SANS were prepared at various surfactant concentrations above the CMC at three different contrasts: h-surfactant in d-choline chloride: d-glycerol, h-surfactant in h-choline chloride:d-glycerol and h-surfactant in $\mathrm{h} / \mathrm{d}$-choline chloride: h/d-glycerol (mole ratios: $0.38 \mathrm{~h}$-choline chloride/0.62 d-choline chloride; 0.56 h-glycerol/0.44 d-glycerol). Equivalent mole fractions of each system were prepared for the three surfactants $\left(\mathrm{C}_{12}-\mathrm{PC}\right.$, SB3-12 and SB3-14) by mixing each protonated surfactant with the solvent. Samples of surfactant mixtures were prepared at one contrast following the same procedure. A homogeneous powder mixture of the protonated surfactants was mixed with d-choline chloride:d-glycerol, at two different concentrations and at the aforementioned surfactant ratios. 
A systematic procedure was used to analyse the data presented here. Data from pure surfactant systems were simultaneously fitted to all three contrasts, whereas the single contrast surfactant mixture data was fitted individually. Three models were initially compared using the Chi Square goodness-of-fit parameter in order to evaluate the best option to fit the intramicellar contribution to the scattering data: monodisperse and polydisperse homogeneous spheres, and monodisperse homogeneous ellipsoids models. ${ }^{46}$ The results from all three models are compared for one of the systems $\left(\mathrm{h}-\mathrm{C}_{12}-\mathrm{PC}\right.$ in 1:2 d-choline chloride:d-glycerol) in the ESI. $\dagger$ The ellipsoid model was shown to provide the best fits to the data and therefore used for the detailed analysis of the data. This model uses the following parameters: equatorial radius or radius of rotation $\left(r_{\mathrm{eq}}\right)$, aspect ratio $\left(\mathrm{AR}=r_{\mathrm{po}} / r_{\mathrm{eq}}\right.$, where $r_{\mathrm{po}}$ is the polar radius of the scatterer), volume fraction of scatterers $\left(\phi_{P(q)}\right)$ and SLD of the solvent and the surfactants. ${ }^{46}$ The radius of gyration of the ellipsoidal scatterers was calculated as follows:

$$
R_{\mathrm{g}}=\frac{\sqrt{r_{\mathrm{eq}}^{2}+r_{\mathrm{po}}^{2}}}{2}
$$

The SLD of the solvent was derived from the results obtained from reflectivity measurements. In terms of surfactant SLDs, the contribution of the headgroup region to the scattering will be considerably reduced due to solvation. Thus the limited contrast between the solvent and the headgroups did not permit a more detailed model of the micelle (e.g. core-shell structure) and so the SLD of the micelle was considered to be that of the surfactant tails. A full record of these values is included in the ESI. $\dagger$ No size polydispersity was included in the model-fitting. The reasons for this are discussed later.

Subsequently, an attempt to simultaneously fit the data using a core-shell ellipsoid model was performed in order to probe the structural heterogeneity of the micelles. However, the low signal-to-noise ratio limited the applicability of this model, more evident at low surfactant concentration where the statistical significance of the results was insufficient. One of the high concentrations was fitted to this model in order to show the core-shell density distribution of the micelle. This was performed following this systematic procedure: the aspect ratio of the aggregate was constrained to that obtained using a uniform ellipsoid model (AR), the thickness of the shell ( $\left.t_{\text {eq,shell }}\right)$ was fitted using the size of the headgroup region from our reflectivity results as the initial guess, and the effective radius was recalculated for the new micelle morphology. A full record of these values is included in the ESI. $\dagger$

Some of the main factors that determine the intermicellar interactions between charged aggregates are the ionic strength and the dielectric constant of the environment. However, the understanding of the ionic behaviour of DES is still not well understood and, although it is know to behave as a polar environment, ${ }^{47}$ those parameters remain rather unexplored. Thus, in order to account for any interaction between the micelles, a structure factor based on a rescaled Percus-Yevick approximation was used. The suitability of this model to account for the intermicellar scattering in DES has been previously discussed. ${ }^{32,33,48}$ This approach modifies the original Hard-Sphere structure factor in order to account for any weak electrostatic repulsion between anisotropic interacting particles. ${ }^{49,50}$ The structure factor model is built using two parameters: the volume fraction of interacting hard spheres $\left(\phi_{S(q)}\right)$ and the radius of interaction $\left(R_{\text {eff }}\right)$. Since the particles slightly deviate from sphericity, a correction for the radius of interaction was applied. The fitting procedure calculates the $R_{\mathrm{eff}}$ as the second virial coefficient of the particle $\left(R_{\mathrm{eff}}=\left(r_{\mathrm{po}} r_{\mathrm{eq}}{ }^{2}\right)^{1 / 3}\right)$ and fits $\phi_{S(q)}{ }^{33,51,52}$

The SANS data was analysed using SasView 4.1. ${ }^{45}$ The modelfitting optimisation was performed using a LevenbergMarquardt algorithm within a $q$-range between 0.007 and $0.5 \AA^{-1}$. The models were smeared using a Gaussian distribution with a constant $\mathrm{d} q / q=5 \%$ in order to account for the instrument resolution.

\section{Results}

\section{Behaviour of pure surfactants}

Zwitterionic surfactants were found to dissolve and remain surface active in choline chloride:glycerol. The surfactant behaviour at the interface is considered to be similar to that in water, where the surfactant diffuses to the surface due to the lyophobic effect. Once the equilibrium at the interface has been reached, the surface tension of the system is also equilibrated, as previously shown for alkyltrimethylammonium bromides in choline chloride:glycerol DES. ${ }^{33}$ In all the cases, the addition of surfactant initially produces a decrease of the surface tension of the system, indicative of the adsorption of surfactant at the interface. Above a certain concentration, the surface of the liquid is saturated with surfactant and further reduction of the surface tension is not observed. This point is the CMC and correlates with the formation of micelles in the continuous phase. The CMC of the pure surfactant systems $\left(\mathrm{C}_{12}-\mathrm{PC}, \mathrm{SB} 3-12\right.$ and SB3-14), the limiting surface tension at the CMC $\left(\gamma_{\mathrm{CMC}}\right)$ and surface pressure at the CMC $\left(\pi=\gamma_{0}-\gamma_{\mathrm{CMC}}\right)$ are presented in Table 1. The surface tension of pure DES $\left(\gamma_{0}=62.9 \pm 0.4 \mathrm{mN} \mathrm{m}^{-1}\right)$ and water $\left(72.4 \pm 0.2 \mathrm{mN} \mathrm{m}^{-1}\right)$ at room temperature were measured as controls and found to be consistent with our previous measurements. ${ }^{33}$

Both headgroup and tail structure were found to affect the CMC. The phosphocholine surfactant shows a lower CMC $(7.9 \pm 0.3 \mathrm{mM})$ than its homologous $\mathrm{C}_{12}$ sulfobetaine, SB3-12 $(14 \pm 2 \mathrm{mM})$. In the case of sulfobetaines, increasing the number

Table 1 CMC, limiting surface tension and surface pressure at the CMC for zwitterionic surfactants in 1:2 choline chloride : glycerol. The values for those surfactants in water are presented for comparison with the results in DES

\begin{tabular}{|c|c|c|c|c|c|c|}
\hline \multirow[b]{2}{*}{ Surfactant } & \multicolumn{2}{|l|}{$\mathrm{CMC} / \mathrm{mM}$} & \multicolumn{2}{|c|}{$\gamma_{\mathrm{CMC}} / \mathrm{mN} \mathrm{m}^{-1}$} & \multicolumn{2}{|l|}{$\pi / \mathrm{mN} \mathrm{m}^{-1}$} \\
\hline & DES & Water & DES & Water & DES & Water \\
\hline $\mathrm{C}_{12}-\mathrm{PC}$ & $7.9 \pm 0.3$ & $0.91^{29}$ & $37.3 \pm 0.2$ & $40.5^{29}$ & $25.6 \pm 0.6$ & $31.9^{29}$ \\
\hline SB3-12 & $14 \pm 2$ & $1.61^{28}$ & $36.7 \pm 0.5$ & $32.79^{28}$ & $26.2 \pm 1.1$ & $39.61^{28}$ \\
\hline SB3-14 & $2.1 \pm 0.2$ & $0.377^{28}$ & $35.0 \pm 0.2$ & $31.84^{28}$ & $27.9 \pm 0.8$ & $40.56^{28}$ \\
\hline
\end{tabular}


of carbons in the surfactant tail from 12 to 14 , decreases the CMC from $14 \pm 2 \mathrm{mM}$ to $2.1 \pm 0.2 \mathrm{mM}$.

Interestingly, this behaviour is very similar to that observed in water for the same surfactants, where the $\mathrm{CMC}$ of $\mathrm{C}_{12}-\mathrm{PC}$ is lower than that of SB3-12. ${ }^{28,29}$ In fact, in both solvents the CMC of $\mathrm{C}_{12}$-PC is about 44\% lower than the CMC of SB3-12. Nonetheless the absolute values of the CMC were found to be consistently lower in water, suggesting higher monomer solubility in DES. An increase in the CMC was previously observed with the addition of ethylene glycol to the aqueous solutions of sulfobetaines. ${ }^{53}$ Those results suggest that the solvophobicity of non-polar moieties is reduced by the presence of ethylene glycol. Similarly, depression of the solvophobic effect has also been previously suggested for cationic surfactants in the same DES. ${ }^{33}$

The limiting surface tension in DES also follows the same trend as in water, although the extent of variation is much less pronounced. The system reaches the lowest value of surface tension at the CMC for SB3-14 and the highest for $\mathrm{C}_{12}$-PC (see Table 1). However, the total variation for these surfactants in DES is only $2.3 \mathrm{mN} \mathrm{m}^{-1}$, as opposed to nearly $8 \mathrm{mN} \mathrm{m}^{-1}$ in water. Actually, this difference is most significant between the PC surfactant and the two SB surfactants. This suggests that the headgroups are affected by the solvent to a different extent.

The structures of surface adsorbed layers of $\mathrm{C}_{12}$-PC and SB3-12 surfactants were measured at the CMC in order to identify any difference in the behaviour of these species at the interface. Fig. 2 shows the XRR data of a pure subphase, $\mathrm{C}_{12}$-PC at its CMC and SB3-12 at its CMC. The results from the best fits are included in Table 2 . A complete record of the fits is included in the ESI. $\dagger$

A bare choline chloride:glycerol surface was initially measured in order to determine its characteristics. The values obtained from the fits are: $\mathrm{SLD}_{\mathrm{s}}=10.8 \times 10^{-6} \AA^{-2}$ and surface
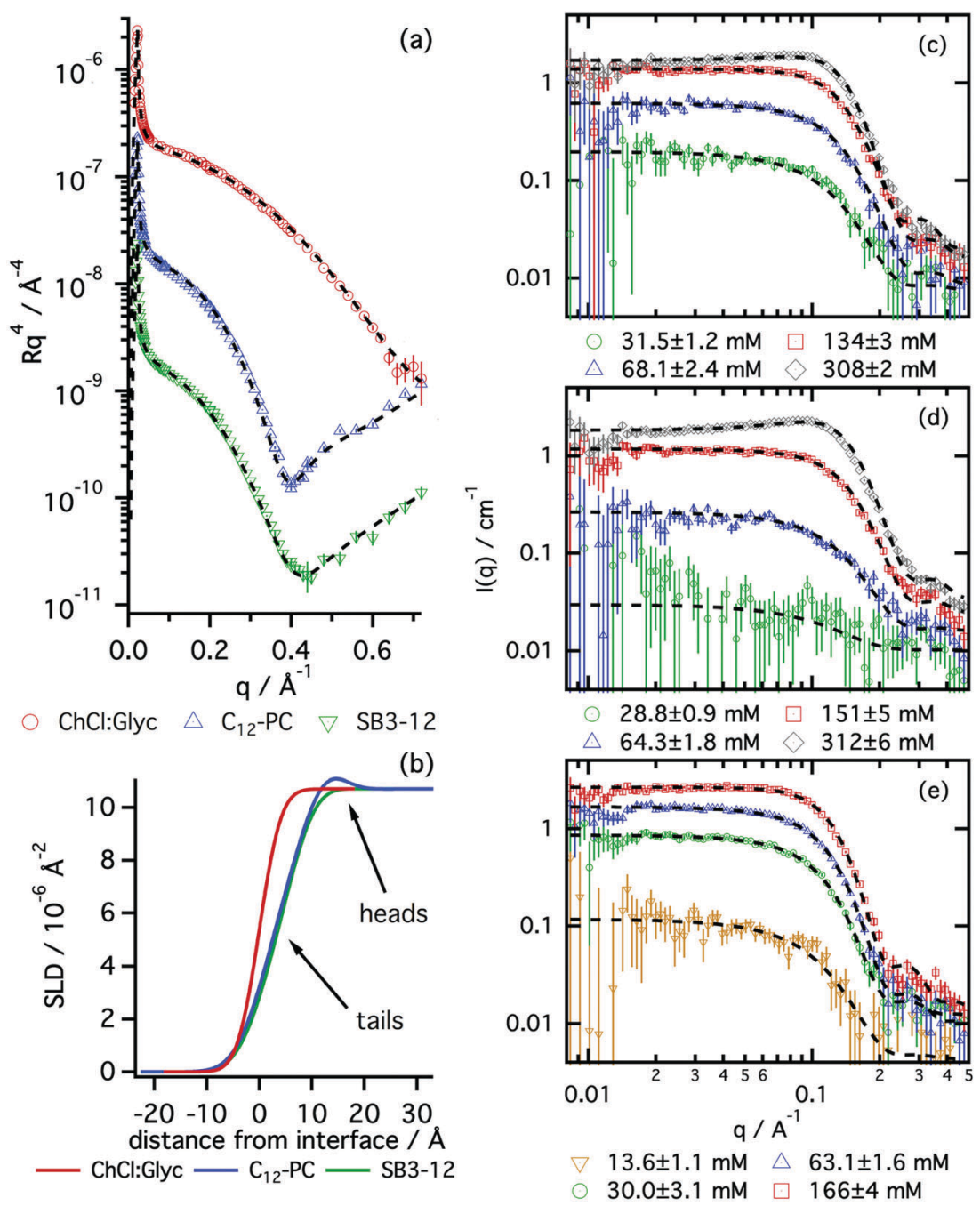

Fig. 2 (a) XRR data (markers) presented as reflectivity $\times q^{4} v s$. $q$ with model fits (black-dashed lines) and (b) SLD profiles of pure choline chloride:glycerol subphase $\mathrm{C}_{12}-\mathrm{PC}$ at the CMC and SB3-12 at the CMC, adsorbed on choline chloride:glycerol DES. SANS data (markers) and best fits (black-dashed lines) of different concentrations of (c) h- $\mathrm{C}_{12}-\mathrm{PC}$, (d) h-SB3-12 and (e) h-SB3-14 in d-choline chloride:d-glycerol. The concentration of surfactant is quoted below each graph. The lowest concentration of SB3-12 could not be fitted following the standard approach and those fits correspond to an approximation of the micelle morphology (obtained from fitting the higher concentrations) at a fitted volume fraction of micelles. 
Table 2 Parameters for the 2-layer plus subphase model used to fit the X-ray reflectivity data presented in Fig. 2

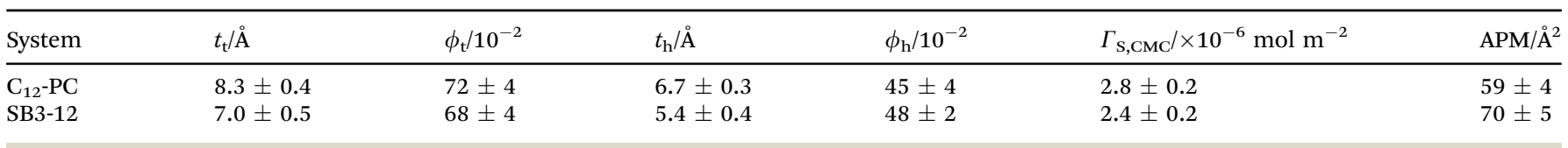

roughness, $\sigma_{\mathrm{s}}=3.3 \AA$, in good agreement with the values previously reported. ${ }^{33}$ These values for the subphase were held fixed during subsequent data fitting from the surfactant systems.

The reflectivity results confirm the adsorption of the pure surfactants to the liquid surface, as both surfactants form a monolayer at the air-liquid interface. $\mathrm{C}_{12}-\mathrm{PC}$ was found to form a thicker monolayer $(15.0 \pm 0.3 \AA)$ than SB3-12 (12.4 $\pm 0.4 \AA)$, at a similar volume fraction of monomer within error. The thicknesses of the surfactant layers in DES were found to be thinner than those in water, where, at the $\mathrm{CMC}, \mathrm{a} \mathrm{C}_{12}-\mathrm{PC}$ monolayer fits to a total thickness of $20 \pm 2 \AA$ and a SB3-12 monolayer shows a total thickness of $14 \pm 3 \AA \AA^{29,54}$ Whereas the thickness of the headgroup layer in DES in both cases is comparable to that in water, ${ }^{40,55}$ thicker monolayers in water may indicate the presence of a stronger lyophobic interaction in water than in DES. The interfaces between different layers appear to be diffuse, potentially due to thermal-induced capillary waves. This effect is reflected in the smearing of the SLD profile on the reflectivity results (see Fig. 2b), as it has been previously reported for SDS on choline chloride:urea. ${ }^{31}$

The surface excess concentration and area per molecule at the interface of these surfactant solutions at the CMC were subsequently calculated from the reflectivity results. The calculations show that the surface excess concentration is higher for the phosphocholine surfactant than for the dodecyl sulfobetaine. Interestingly this is the opposite behaviour found for these amphiphiles in water. Neutron reflectivity results have shown that these surfactants on water, at the CMC, present a surface excess concentration of $3.35 \times 10^{-6} \mathrm{~mol} \mathrm{~m} \mathrm{~m}^{-2}$ and $3.70 \times 10^{-6} \mathrm{~mol} \mathrm{~m}^{-2}$ for $\mathrm{C}_{12} \mathrm{PC}$ and $\mathrm{SB} 3-12$ respectively. ${ }^{29,54}$ The higher values found in water are again indicative of the greater affinity of the DES for solvated surfactant free monomers, which thus leads to reduced surface excess concentrations. Finally, the calculated values indicate that the area per surfactant monomer at the air-DES interface is larger in the case of SB3-12 than $\mathrm{C}_{12}$-PC. This effect is, again, the opposite to the behaviour seen in water, where the area per molecule is slightly larger for $\mathrm{C}_{12}$-PC than for SB3-12.

As anticipated by the results from surface tension, these surfactants were found to aggregate in solution. The morphology and behaviour of those micelles were investigated by means of SANS. Fig. 2 shows the scattering data at one contrast of the three zwitterionic surfactants in choline chloride:glycerol together with the best model fits. The results from those fits are presented in Fig. 3. A full record of the results from the fits is included in the ESI, $\uparrow$ together with the plots for all the SANS contrasts.

All the surfactants presented here were found to form globular micelles above the CMC in DES, with a certain amount

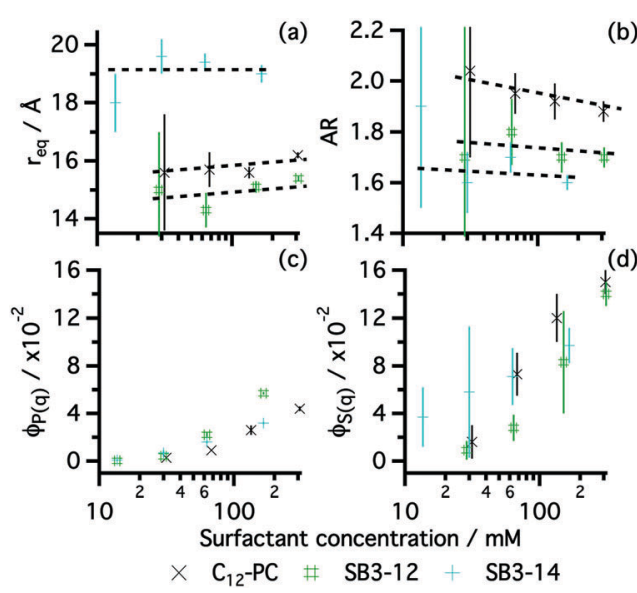

Fig. 3 Fitting parameters derived from the SANS data at different concentrations of $\mathrm{C}_{12}-\mathrm{PC}, \mathrm{SB} 3-12$ and SB3-14 in choline chloride:glycerol: (a) equatorial radius, (b) aspect ratio, (c) $P(q)$ volume fraction and (d) $S(q)$ volume fraction. The lines represent the linear trend of those values.

of intermicellar interaction. The uniform ellipsoid with a prolate distribution of mass (AR > 1) accurately represents the morphology of those micelles, reflected in the good agreement between the models and the experimental data (see Fig. 2c-e).

Previous investigations have used either polydisperse spheres and monodisperse ellipsoids to describe the structure of zwitterionic micelles in water. ${ }^{26,30}$ Our decision, based on both thermodynamic reasons and quality of the fits, was to use the monodisperse ellipsoidal model. As shown by Tanford, the formation of globular micelles with a certain deviation from sphericity constitutes a suitable scenario in terms of micelle morphology, as it would optimise the tail packing in the hydrocarbon core and the entropic contribution. ${ }^{56}$ Furthermore, it has been recently reported that surfactant aggregates, in thermodynamic equilibrium, show little polydispersity in DES. ${ }^{48}$ Therefore no polydispersity function was implemented in our model, as a realistic fit could be obtained without this extra parameter.

Our results show that the structure of the aggregates depends on the tail length and surfactant headgroup. As expected, SB3-14 shows bigger micelles than the SB3-12 driven by the presence of a larger hydrophobic moiety in the surfactant. SB3-14 micelles have a similar size to those in water $\left(R_{g}=19.1 \pm 1 \AA\right),{ }^{57}$ whereas $\mathrm{C}_{12}$-PC micelles were found to be slightly smaller in DES. ${ }^{30}$ Both $\mathrm{C}_{12}$ surfactants show a similar equatorial radius and differences in the AR are small. Although the resolution of the experiment and the low signal-to-noise ratio did not allow the use of a more detailed model of the micelles, an underlying trend can be found in the AR of these surfactants. SB3-12 forms slightly shorter micelles, associated with a smaller AR, potentially driven by the interactions between headgroups at the micelle interface. 
Since the hydrophobic moiety of both $\mathrm{C}_{12}$ surfactants is identical, changes in the packing parameter $\left(v / a_{0} l_{c}\right.$, where $v$ is the volume of the lyophobic moiety, $a_{0}$ is the area at the headgroup-tail interface and $l_{\mathrm{c}}$ is the length of the fully extended tail) are strictly driven by differences in the area at the headgroup-tail interface. ${ }^{58}$ Assuming that geometrical effects will not change the trend in the area per monomer between the planar interface of the monolayer and the curved micelle interface, larger APM would imply larger $a_{0}$. Thus, a larger AR would be expected for $\mathrm{C}_{12}$-PC than for SB3-12. These results therefore correlate with those obtained from reflectivity, where the area per monomer at the interface was found to be larger for SB3-12 than for $\mathrm{C}_{12}$-PC.

From the simultaneous fit of the three contrasts to the coreshell ellipsoid model, detailed structural information about the aggregate could be obtained (see ESI $\dagger$ ). For the three surfactants, a model using a micelle core containing surfactant tails surrounded by solvated headgroups satisfactorily fitted the data. The core of $\mathrm{C}_{12}$-PC and SB3-12 were found to be the same within error (14 $\pm 1 \AA$ ), whereas SB3-14 showed a larger lyophobic core $(19 \pm 1 \AA$ ). Shell thickness was found to vary with the headgroup of the surfactant, in agreement with the reflectivity results, where the phosphocholine headgroup region is thicker than that of the sulfobetaine surfactants (7 $\pm 1 \AA$ for $\mathrm{C}_{12}$-PC, $5 \pm 1 \AA$ for SB3-12 and SB3-14). Also, the volume fraction of the micelle headgroup $\left(\phi_{\mathrm{hg}}\right)$ was found to be consistently smaller than that at the air-liquid interface, obtained through reflectivity $\left(\phi_{\mathrm{h}}\right)$, which potentially causes the different arrangement of surfactant monomers (planar geometry at the interface, globular geometry at the bulk phase). However, the contrast resolution and limited signal-to-noise ratio did not allow further information about the characteristics of this region to be extracted.

Zwitterionic surfactants in pure water show an overall neutral charge, thus long-range electrostatic interactions between micelles are negligible. ${ }^{30}$ Meanwhile, intermicellar interactions in DES are not expected to appear until high surfactant concentration since the solvent has inherently high ionic strength. ${ }^{32,33}$ However, here we have found that hard-sphere interactions were insufficient to account for the intermicellar scattering, and therefore, intermicellar interactions must have a role in these systems. Fig. 3 indexes both the form and structure factor volume fractions as a function of the surfactant concentration. Our results show the clear decoupling between $\phi_{S(q)}$ and $\phi_{P(q)}$, where the contribution from micelle-micelle interactions consistently shows higher volume fractions than those from the intramicellar scattering. These differences may be related to the excess contribution arising from electrostatic interactions between the aggregates, commonly found in simple ionic surfactants but uncommon for zwitterionics in pure water.

We consistently find that the structure factor contribution from $\mathrm{C}_{12}$-PC micelles is greater than those for SB3-12. This may be indicative of differences in ion binding to the headgroup with varying charge distribution in the headgroup. This would therefore create a surface higher charge density by unbalancing the charge neutralisation within the headgroup and/or by affecting the solvent structure surrounding the micelle. ${ }^{59,60}$ Unfortunately, due to the limited physicochemical information of the solvent and instrument resolution, more information about this interaction cannot be extracted from the data presented here.

\section{Surfactant mixtures in choline chloride:glycerol}

The behaviour of mixtures of the $\mathrm{C}_{12}$ phosphocholine and sulfobetaine surfactants was investigated at different molar ratios of surfactant: $\mathrm{C}_{12}$-PC/SB3-12: $0.2 / 0.8,0.35 / 0.65,0.5 / 0.5$, $0.65 / 0.35$ and $0.8 / 0.2$. Surface tension measurements were used to find the $\mathrm{CMC}$ of the systems and elucidate the nature of mixing of the two surfactants $\left(\mathrm{C}_{12}-\mathrm{PC}\right.$ and SB3-12). The surface tension plots of these systems are included in the ESI $\dagger$ and the CMC results are presented in Fig. 4 as a function of the mole fraction of $\mathrm{C}_{12}$-PC in the surfactant mixture.

As seen for the pure surfactant systems, there is a decrease of surface tension with increasing surfactant concentration. The inflexion point indicates the limiting surface tension allowing the CMC to be identified for the various mixtures. When considering ideal mixing of surfactants, a theoretical determination of the CMCs of the mixed systems can be obtained using the pseudophase separation model: ${ }^{61}$

$$
\frac{1}{\mathrm{CMC}_{m}}=\frac{x_{1}}{\mathrm{CMC}_{1}}+\frac{1-x_{1}}{\mathrm{CMC}_{2}}
$$

where $\mathrm{CMC}_{m}, \mathrm{CMC}_{1}$ and $\mathrm{CMC}_{2}$ are the $\mathrm{CMC}$ of the mixture, component 1 and 2 , respectively. $x_{1}$ corresponds to the mole fraction of the component 1 in the surfactant mixture. The results from this calculation are plotted together with the experimental values for comparison (see Fig. 4).

Interactions between the two surfactants when mixed will lead to deviations from the ideal case. Attractive interactions between the amphiphiles lead to a decrease in the experimental values of the CMC, whereas repulsive interactions show higher CMC values for the mixtures. The application of the regular solution theory approximation accounts for the non-ideality of the mixture through modifications in the activity coefficient of each surfactant in the mixture. Following the procedure introduced by Holland and Rubingh, ${ }^{62}$ the interaction between

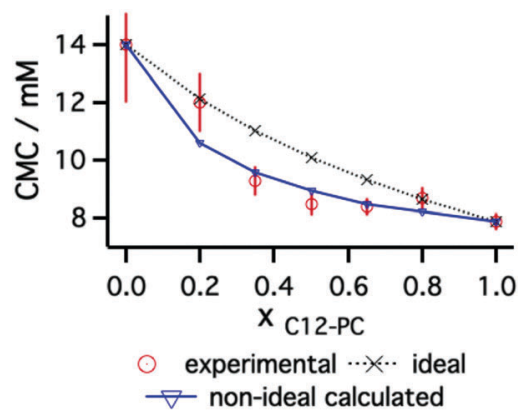

Fig. 4 Experimental, ideal (calculated through the pseudophase separation model) and non-ideal (calculated through the regular solution theory) values of the CMCs of the mixtures at different mole ratios of surfactant. 
surfactants within a binary mixture can be described using a parameter $\beta$, for activity coefficients $f_{1}$ and $f_{2}$ :

$$
\begin{aligned}
\frac{1}{\mathrm{CMC}_{m}} & =\frac{x_{1}}{f_{1} \mathrm{CMC}_{1}}+\frac{1-x_{1}}{f_{2} \mathrm{CMC}_{2}} \\
\ln f_{1} & =\beta\left(1-x_{1}\right)^{2} \\
\ln f_{2} & =\beta x_{1}^{2}
\end{aligned}
$$

Solving the equation iteratively, a $\beta$ value can be calculated for the mixture. Our results show that the $\mathrm{CMC}_{m}$ of the mixtures falls below the ideal value, with $\beta=-0.36$. This suggests that an electrostatic attractive interaction exists between the surfactant monomers in the mixture, leading to deviations from ideality. The regular solution theory model is also represented in Fig. 4.

The interfacial behaviour of the mixtures of zwitterionic surfactants was further probed using X-ray reflectivity.
Data and best fits are presented in Fig. 5, and results from those fits are summarised in Fig. 6. A complete record of the fitting results is included in the ESI. $\dagger$

Mixed monolayers at the CMC show a similar structure to those of pure surfactants: a dry tail region and a solvated headgroup layer. The dimensions of the various layers change with the ratio of each surfactant in the mixture. At the CMC, the monolayer total thickness gradually varies from thinner values at low $\mathrm{C}_{12}$-PC mole fractions to thicker monolayers at high $\mathrm{C}_{12}$-PC mole fractions. These results fit the trend established for the pure surfactants at the interface, for which the $\mathrm{C}_{12}$-PC monolayer is thicker than that of pure SB3-12. The area per molecule also gradually varies, showing a decrease with increasing amount of $\mathrm{C}_{12}$-PC. Those values were found to negatively deviate from the ideal values, suggesting that mixed species allow tighter packing potentially due to attractive interactions between monomers.

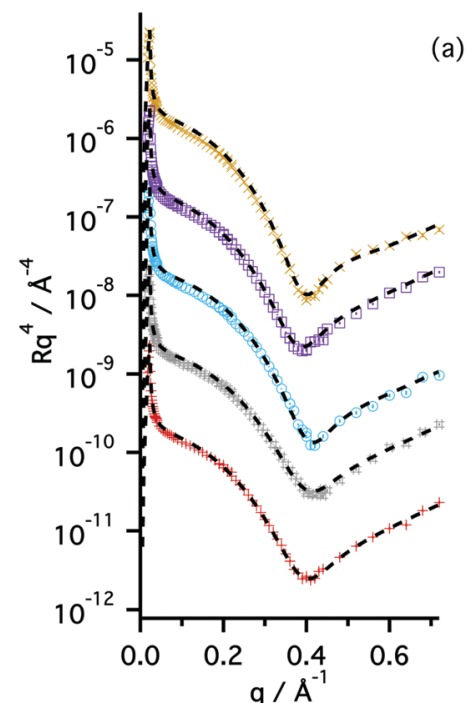

(a)
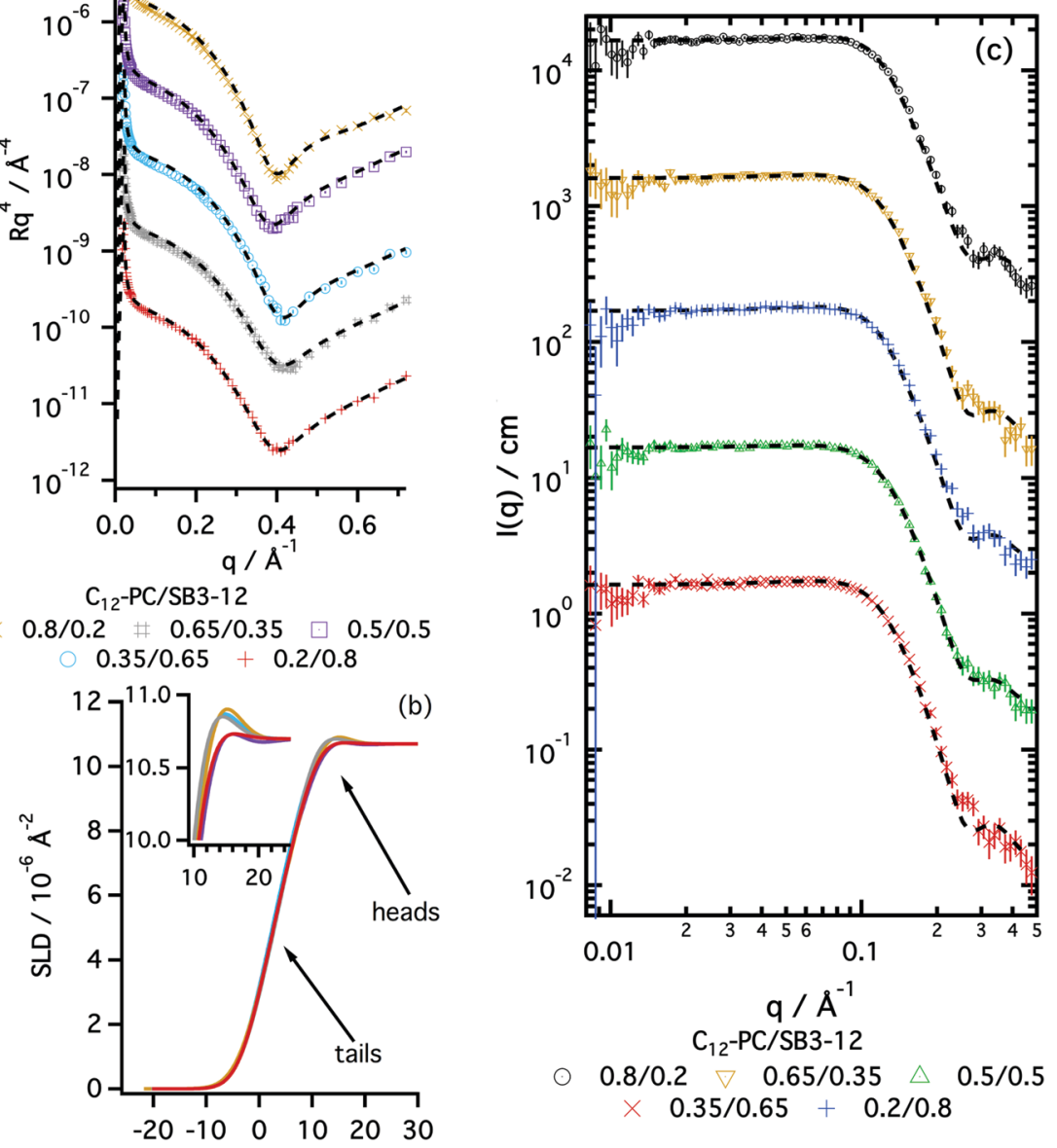

distance from interface / $\AA$ $\mathrm{C}_{12}-\mathrm{PC} / \mathrm{SB} 3-12$

$-0.8 / 0.2-0.65 / 0.35-0.5 / 0.5$

$0.35 / 0.65-0.2 / 0.8$

Fig. 5 (a) XRR data (markers) presented as reflectivity $\times q^{4}$ vs. $q$ with model fits (black-dashed lines) and (b) SLD profiles of different mole fractions of $\mathrm{C}_{12}$-PC/ SB3-12 in the surfactant mixtures on a choline chloride:glycerol subphase. The inset in (b) is a magnification of the headgroup region of the SLD profile. (c) SANS data (markers) and best fits (black-dashed lines) of different mixtures of $\mathrm{C}_{12}$-PC/SB3-12 surfactants at a total surfactant concentration of $185 \pm 3$ mM. The mole fractions of $\mathrm{C}_{12}-\mathrm{PC}$ in the surfactant mixture are presented in the legend of the graph. The intensity of data and fits has been offset for clarity. 


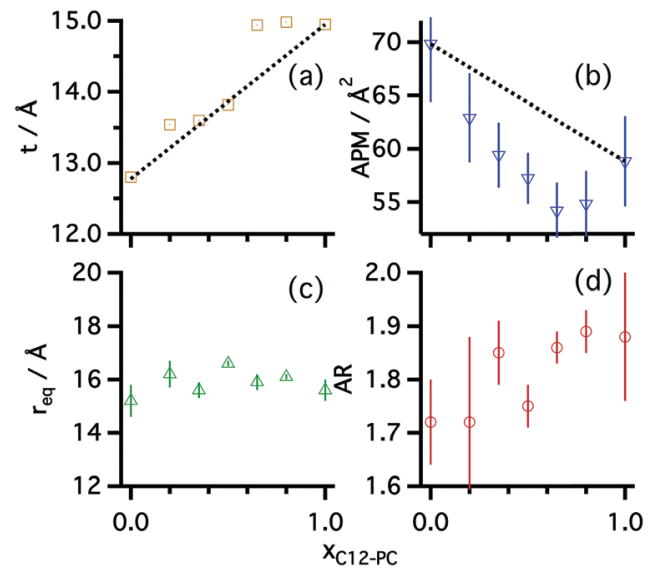

Fig. 6 (a) Monolayer thickness and (b) area per surfactant molecule of mixtures of $\mathrm{C}_{12}-\mathrm{PC}$ and SB3-12 at the air-liquid interface. The areas per molecule are compared to the ideal values obtained from a simple average of the values of pure surfactants (black-dotted lines). Variation of (c) equatorial radius and (d) AR of the mixed micelles in choline chloride:glycerol at different mole fractions of $\mathrm{C}_{12}-\mathrm{PC}: \mathrm{SB} 3-12$ at the total surfactant concentration of $185 \pm 3 \mathrm{mM}$. Values for pure surfactants are included for comparison $\left(x_{C_{12}-\mathrm{PC}}=0\right.$ and 1$)$

The formation of micelles by these surfactant mixtures in DES was investigated by means of SANS. Two different concentrations of the mixtures were measured above the CMC in a single contrast and fitted using the homogeneous ellipsoid model. Fig. 5 shows the SANS data and best fits of the different h-surfactant mixtures in 1:2 d-choline chloride : d-glycerol. The results from the fits are summarised in Fig. 6 and a full record of the fitted parameters is included in the ESI. $\dagger$

As shown for the systems containing pure zwitterionic surfactants in choline chloride:glycerol, the agreement between the data and mathematical model demonstrates that prolate ellipsoid is a suitable model to represent zwitterionic mixed micelles in DES. The variation of surfactant mole fraction in the surfactant mixture leads to subtle changes in the morphology of the aggregates. Whereas the equatorial radius remains practically unchanged as the composition changes, the AR of the micelles gradually evolves with the molar ratio. These results indicate a variation in the area per surfactant monomer at the micelle interface and match the findings from XRR: increasing the amount of $\mathrm{C}_{12}$-PC in the mixture leads to a smaller area per surfactant monomer, and therefore promotes the formation of more elongated aggregates (see Fig. $6 \mathrm{~b}$ and $\mathrm{d}$ ).

The volume fractions of the mixed micelles extracted from the structure factor and the form factor were found to behave in a similar manner to those of the pure systems. Increasing the total volume fraction of micelles in solution leads to a considerable increase of the apparent $S(q)$ volume fraction, the latter being considerably higher than the volume fraction of micelles ( $\sim 3$ times higher at $185 \pm 3 \mathrm{mM}$ surfactant concentration). Due to systematic variability between samples it is however difficult to draw conclusions about the $S(q)$ evolution as the composition of the mixture is varied.

\section{Discussion}

Zwitterionic surfactants were found to preserve their activity in choline chloride:glycerol DES. The solvation of zwitterionic moieties becomes highly complex when it occurs in DES. The presence of charged ions in the solvent and neutral compounds with H-bonding capability may promote the formation of solvating environments, where the solvent components are segregated. ${ }^{12,19,32,33}$ The presence of positively charged choline ions in close proximity to the sulfate or phosphate group may be favoured due to electrostatic interactions and H-bonding. Moreover, the choline group of the surfactant heads may favour the presence of glycerol in its local solvation environment. ${ }^{19,33}$ Such considerations could lead to the formation of solvation layers that would affect the behaviour of the amphiphiles and, ultimately, the morphology of the resultant aggregates. The effect of solvent layering has been explored and confirmed for the nanoparticles in DES, where electrostatics govern the formation of distinct choline-rich or hydrogen bond donor-rich layers surrounding the particles. ${ }^{60,63,64}$

Surface tension results have shown differences in the CMC values between surfactants indicating differences in the solvation of the sulfobetaine and phosphocholine headgroups. These differences were corroborated by our XRR and SANS results. In both cases the micelles are globular and the surfactants show a relatively high resilience to the high ionic strength of the solvent, in that the morphologies are globular and therefore not profoundly altered compared to the analogous systems in water. This is in notable contrast to anionic surfactants in choline chloride:urea or cationic surfactants in choline chloride:malonic acid. ${ }^{13,32}$

As occurs in water, the presence of salts at the interface also promotes changes in the monomer-monomer electrostatic interactions, as widely seen for phospholipid monolayers and bilayers. ${ }^{22,40,55,65,66}$ Interestingly, such ion-ion interactions were reported to be more pronounced when counterions interact with the charged group adjacent to the tail than with the terminal group. ${ }^{40,65,66}$ Although the surfactants investigated here both have positive and negative charges, the relative position of those charges in the headgroup seem to alter the structure of the aggregates, presumably through modifications to the monomer packing or solvation shell. Unfortunately, limited resolution and SLD contrast (due to solvation) in the scattering data mean we cannot determine a more detailed structure of the headgroup and solvation shell of the micelles or monolayers.

Charge screening has been found to be a significant influence on the intermicellar interaction of anionic surfactants in DES. This was explained through the ion-pair formation between solvent cations and surfactant native counterions from the solvent and the anionic headgroups. ${ }^{32,35}$ However, cationic surfactants in the same solvent showed a stronger interaction between micelles. This was attributed to the weaker effect of chloride/bromide anions binding to the surfactant headgroups. ${ }^{33}$

Zwitterionic micelles also show intermicellar interactions that depend on the characteristics of the headgroup. The phosphocholine surfactant consistently showed higher structure factor 
volume fractions, an indicator of intermicellar repulsion, than its sulfobetaine analogue. These findings could be interpreted in two ways. Traditionally, long-range electrostatic interactions have been associated to the ionic character of the aggregates. ${ }^{67}$ In this case, DES may somehow behave as a relatively low ionic strength environment, where the electrostatic interactions are not totally screened. This correlates with results suggesting that ionic liquids behave as relatively dilute electrolytes and long-range electrostatic interactions are retained. ${ }^{68}$ The excess interactions observed here may be explained through partial adsorption of ions to the headgroups. In pure water, zwitterionic headgroups can be considered as neutral moieties, ${ }^{30}$ so the formation of ion-pairs between the solvent and one of the charges in the headgroup in DES could explain the differences between in behaviour in these solvents. In a second plausible scenario, the presence of charged macromolecules in DES may influence the structure of the solvent surrounding the micelle, producing a segregated-solvent superstructure that will finally affect the intermicellar interactions and, thus, the structure factor contained in the scattering data. This has been previously reported for nanoparticles in DES, ${ }^{60,63,69}$ where the surface charge of the particles produce a re-arrangement of the solvent surrounding the particle through the charge imbalance at the interface. Similarly, the net charge showed by the micelles could promote a similar behaviour. However, the instrument and contrast resolution of the data presented here is insufficient to probe that.

The results from surfactant mixtures indicate that ionion interactions between sulfobetaine and phosphocholine headgroups affect the surface and bulk behaviour of the amphiphiles. Investigations of the aqueous behaviour of mixed phosphocholine/sulfobetaine monolayers showed a negative deviation from ideality of the APM values using the additivity rule. ${ }^{36}$ Similarly, surface tension and reflectivity demonstrates a non-ideal mixing of the surfactants, probably influenced by Coulombic attraction between different headgroups, resulting in smaller CMCs and average molecular areas. Variations in the APM calculated through reflectivity are hypothesised to be similar to those at the micelle interface, such that a smaller APM at the interface can be extrapolated to smaller areas at the micelle interface and therefore larger ARs of the micelles. The SANS results indeed showed that variations in micelle AR are present as the composition of the mixture was varied, with slightly more elongated micelles formed at ratios which correlated to smaller APMs.

\section{Conclusions}

Zwitterionic surfactants have been demonstrated to self-assemble in choline chloride:glycerol. The surface activity is retained as shown by surface tension, and the CMC of the system depends on the chain length of the surfactant and the headgroup. Increasing the chain length from $\mathrm{C}_{12}$ to $\mathrm{C}_{14}$ in the case of the sulfobetaine results in a decrease of the CMC. Furthermore, a significant difference in the CMC with different headgroups was found, showing a higher CMC for the sulfobetaine than for the phosphocholine surfactant, comparable to that which occurs in water. These differences result from changes in the headgroup structure and are confirmed by structural investigations of the monolayer by means of X-ray reflectivity. The reflectivity results demonstrate variations in the monolayer structure, with differences in the area per molecule and thickness of the layers. SB3-12 shows larger area per molecule at the $\mathrm{CMC}$ than $\mathrm{C}_{12}$-PC.

Above the CMC, pure surfactants were found to form micelles in the bulk phase. SB3-12 forms micelles with a smaller AR than those of $\mathrm{C}_{12}$-PC. Unsurprisingly, SB3-14 was found to form bigger micelles than its $\mathrm{C}_{12}$ analogue. Intermicellar interaction appears to be stronger for $\mathrm{C}_{12}-\mathrm{PC}$ surfactant, suggesting differences in the solvation and charge screening at this surfactant headgroup. Mixtures of surfactants were found to behave similarly to the pure surfactants, also forming globular micelles. Surface tension and reflectivity results showed a subtle negative deviation from ideal behaviour of the mixture. Modelling of those results confirm that such deviations arise from electrostatic attractive interactions between neighbouring headgroups.

These investigations demonstrate the activity and aggregation of zwitterionic amphiphiles in choline chloride:glycerol DES, with certain similarities to the behaviour already known in water. Interestingly, this DES has been found to effectively solvate the headgroup of the surfactants, although the solvation mechanism of these moieties still remains unclear. Future investigations to explore such phenomena could include isotopic-substitution small-angle neutron scattering and neutron reflectivity. These results will potentially lead to the development of a framework to facilitate new methods of drugdelivery and biosensing technologies, as well as to better understand the formation of phospholipid membranes and the conformation of proteins in DES.

\section{Conflicts of interest}

There are no conflicts of interest to declare.

\section{Acknowledgements}

The authors would like to acknowledge Diamond Light Source and the ISIS Pulsed Neutron and Muon Source for the award of beamtime on the I07 and LOQ instruments (experiment numbers SI15584-1 and RB1710047, respectively). We further thank Diamond Light Source for the access to the drop-shape analysis instrument. A. S.-F. would like to thank the University of Bath Alumni Fund and the European Spallation Source for funding his PhD studentship. We gratefully acknowledge the University of Bath for supporting G. M., L. C. M. thanks EPSRC for a DTP studentship. This work benefited from the use of the SasView application, originally developed under NSF Award DMR-0520547. SasView also contains code developed with funding from the EU Horizon 2020 programme under the SINE2020 project Grant No. 654000. 


\section{References}

1 E. L. Smith, A. P. Abbott and K. S. Ryder, Chem. Rev., 2014, 114, 11060-11082.

2 A. Paiva, R. Craveiro, I. Aroso, M. Martins, R. L. Reis and A. R. C. Duarte, ACS Sustainable Chem. Eng., 2014, 2, 1063-1071.

3 Y. Dai, J. van Spronsen, G. J. Witkamp, R. Verpoorte and Y. H. Choi, Anal. Chim. Acta, 2013, 766, 61-68.

4 A. Hayyan, F. S. Mjalli, I. M. AlNashef, T. Al-Wahaibi, Y. M. Al-Wahaibi and M. A. Hashim, Thermochim. Acta, 2012, 541, 70-75.

5 Q. Zhang, K. De Oliveira Vigier, S. Royer and F. Jerome, Chem. Soc. Rev., 2012, 41, 7108-7146.

6 O. S. Hammond, D. T. Bowron and K. J. Edler, Green Chem., 2016, 18, 2736-2744.

7 O. S. Hammond, D. T. Bowron and K. J. Edler, Angew. Chem., Int. Ed., 2017, 56, 9782-9785.

8 D. V. Wagle, G. A. Baker and E. Mamontov, J. Phys. Lett., 2015, 6, 2924-2928.

9 C. F. Araujo, J. A. P. Coutinho, M. M. Nolasco, S. F. Parker, P. J. A. Ribeiro-Claro, S. Rudic, B. I. G. Soares and P. D. Vaz, Phys. Chem. Chem. Phys., 2017, 19, 17998-18009.

10 B. Tang and K. H. Row, Monatsh. Chem., 2013, 144, 1427-1454.

11 R. Esquembre, J. M. Sanz, J. G. Wall, F. del Monte, C. R. Mateo and M. L. Ferrer, Phys. Chem. Chem. Phys., 2013, 15, 11248-11256.

12 S. J. Bryant, R. Atkin and G. G. Warr, Langmuir, 2017, 33, 6878-6884.

13 A. Sanchez-Fernandez, K. J. Edler, T. Arnold, D. Alba Venero and A. J. Jackson, Phys. Chem. Chem. Phys., 2017, 19, 8667-8670.

14 B.-P. Wu, Q. Wen, H. Xu and Z. Yang, J. Mol. Catal. B: Enzym., 2014, 101, 101-107.

15 A. A. Papadopoulou, E. Efstathiadou, M. Patila, A. C. Polydera and H. Stamatis, Ind. Eng. Chem. Res., 2016, 55, 5145-5151.

16 A. R. Harifi-Mood, R. Ghobadi and A. Divsalar, Int. J. Biol. Macromol., 2017, 95, 115-120.

17 R. P. Xin, S. J. Qi, C. X. Zeng, F. I. Khan, B. Yang and Y. H. Wang, Food Chem., 2017, 217, 560-567.

18 I. Mamajanov, A. E. Engelhart, H. D. Bean and N. V. Hud, Angew. Chem., 2010, 122, 6454-6458.

19 S. J. Bryant, R. Atkin and G. G. Warr, Soft Matter, 2016, 12, 1645-1648.

20 F. Milano, L. Giotta, M. R. Guascito, A. Agostiano, S. Sblendorio, L. Valli, F. M. Perna, L. Cicco, M. Trotta and V. Capriati, ACS Sustainable Chem. Eng., 2017, 5, 7768-7776.

21 H. Mohwald, Annu. Rev. Phys. Chem., 1990, 41, 441-476.

22 R. Ekerdt and D. Papahadjopoulos, Proc. Natl. Acad. Sci. U. S. A., 1982, 79, 2273-2277.

23 O. Choksupmanee, K. Hodge, G. Katzenmeier and S. Chimnaronk, Biochemistry, 2012, 51, 2840-2851.

24 X. Wang, K. Corin, C. Rich and S. Zhang, Sci. Rep., 2011, 1, 102.

25 B. L. Cook, D. Steuerwald, L. Kaiser, J. Graveland-Bikker, M. Vanberghem, A. P. Berke, K. Herlihy, H. Pick, H. Vogel and S. Zhang, Proc. Natl. Acad. Sci. U. S. A., 2009, 106, 11925-11930.
26 J. Lipfert, L. Columbus, V. B. Chu, S. A. Lesley and S. Doniach, J. Phys. Chem. B, 2007, 111, 12427-12438.

27 J. P. Priebe, M. L. Satnami, D. W. Tondo, B. S. Souza, J. M. Priebe, G. A. Micke, A. C. O. Costa, H. D. Fiedler, C. A. Bunton and F. Nome, J. Phys. Chem. B, 2008, 112, 14373-14378.

28 G. Qu, J. Cheng, J. Wei, T. Yu, W. Ding and H. Luan, J. Surfactants Deterg., 2011, 14, 31-35.

29 M. Yaseen, Y. Wang, T. J. Su and J. R. Lu, J. Colloid Interface Sci., 2005, 288, 361-370.

30 E. Pambou, J. Crewe, M. Yaseen, F. N. Padia, S. Rogers, D. Wang, H. Xu and J. R. Lu, Langmuir, 2015, 31, 9781-9789.

31 T. Arnold, A. J. Jackson, A. Sanchez-Fernandez, D. Magnone, A. E. Terry and K. J. Edler, Langmuir, 2015, 31, 12894-12902. 32 A. Sanchez-Fernandez, K. J. Edler, T. Arnold, R. K. Heenan, L. Porcar, N. J. Terrill, A. E. Terry and A. J. Jackson, Phys. Chem. Chem. Phys., 2016, 18, 14063-14073.

33 A. Sanchez-Fernandez, T. Arnold, A. J. Jackson, S. L. Fussell, R. K. Heenan, R. A. Campbell and K. J. Edler, Phys. Chem. Chem. Phys., 2016, 18, 33240-33249.

34 A. P. Abbott, R. C. Harris, K. S. Ryder, C. D'Agostino, L. F. Gladden and M. D. Mantle, Green Chem., 2011, 13, 82-90.

35 A. Sanchez-Fernandez, O. S. Hammond, K. J. Edler, T. Arnold, J. Doutch, R. M. Dalgliesh, P. Li, K. Ma and A. J. Jackson, Phys. Chem. Chem. Phys., 2018, 20, 13952-13961.

36 T. Aikawa, K. Yokota, T. Kondo and M. Yuasa, Langmuir, 2016, 32, 10483-10490.

37 T. Arnold, C. Nicklin, J. Rawle, J. Sutter, T. Bates, B. Nutter, G. McIntyre and M. Burt, J. Synchrotron Radiat., 2012, 19, 408-416.

38 A. Nelson, J. Appl. Crystallogr., 2006, 39, 273-276.

39 F. Abelès, J. Phys. Radium, 1950, 11, 307-309.

40 G. Hazell, A. P. Gee, T. Arnold, K. J. Edler and S. E. Lewis, J. Colloid Interface Sci., 2016, 474, 190-198.

41 L. Braun, M. Uhlig, R. von Klitzing and R. A. Campbell, Adv. Colloid Interface Sci., 2017, 247, 130-148.

42 R. K. Heenan, J. Penfold and S. M. King, J. Appl. Crystallogr., 1997, 30, 1140-1147.

43 O. Arnold, J. C. Bilheux, J. M. Borreguero, A. Buts, S. I. Campbell, L. Chapon, M. Doucet, N. Draper, R. Ferraz Leal, M. A. Gigg, V. E. Lynch, A. Markvardsen, D. J. Mikkelson, R. L. Mikkelson, R. Miller, K. Palmen, P. Parker, G. Passos, T. G. Perring, P. F. Peterson, S. Ren, M. A. Reuter, A. T. Savici, J. W. Taylor, R. J. Taylor, R. Tolchenov, W. Zhou and J. Zikovsky, Nucl. Instrum. Methods Phys. Res., Sect. A, 2014, 764, 156-166.

44 G. D. Wignall and F. S. Bates, J. Appl. Crystallogr., 1987, 20, 28-40. 45 M. Doucet, J. H. Cho, G. Alina, J. Bakker, W. Bouwman, P. Butler, K. Campbell, M. Gonzales, R. Heenan, A. Jackson, P. Juhas, S. King, P. Kienzle, J. Krzywon, A. Markvardsen, T. Nielsen, L. O'Driscoll, W. Potrzebowski, R. Ferraz Leal, T. Richter, P. Rozycko and A. Washington, 2017, DOI: 10.5281/zenodo.438138.

46 J. S. Pedersen, Adv. Colloid Interface Sci., 1997, 70, 171-210. 47 A. Pandey, R. Rai, M. Pal and S. Pandey, Phys. Chem. Chem. Phys., 2014, 16, 1559-1568. 
48 A. Sanchez-Fernandez, O. S. Hammond, A. J. Jackson, T. Arnold, J. Doutch and K. J. Edler, Langmuir, 2017, 33, 14304-14314.

49 J. A. Barker and D. Henderson, Rev. Mod. Phys., 1976, 48, 587-671.

50 D. J. Kinning and E. L. Thomas, Macromolecules, 1984, 17, 1712-1718.

51 Y. C. Liu, S. H. Chen and R. Itri, J. Phys.: Condens. Matter, 1996, 8, A169-A187.

52 V. K. Aswal and P. S. Goyal, Chem. Phys. Lett., 2003, 368, 59-65.

53 M. del Mar Graciani, A. Rodríguez, M. Muñoz and M. L. Moyá, Langmuir, 2005, 21, 7161-7169.

54 P. Li, K. Ma, R. K. Thomas and J. Penfold, J. Phys. Chem. B, 2016, 120, 3677-3691.

55 M. Yaseen, J. R. Lu, J. R. P. Webster and J. Penfold, Biophys. Chem., 2005, 117, 263-273.

56 A. Ben-Shaul, I. Szleifer and W. M. Gelbart, Proc. Natl. Acad. Sci. U. S. A., 1984, 81, 4601-4605.

57 J. A. Holdaway, PhD thesis, University of Bath, 2013.

58 J. N. Israelachvili, D. J. Mitchell and B. W. Ninham, J. Chem. Soc., Faraday Trans. 2, 1976, 72, 1525.

59 M. d. S. Baptista, I. Cuccovia, H. Chaimovich, M. J. Politi and W. F. Reed, J. Phys. Chem., 1992, 96, 6442-6449.
60 J. A. Hammons, F. Zhang and J. Ilavsky, J. Colloid Interface Sci., 2018, 520, 81-90.

61 J. H. Clint, J. Chem. Soc., Faraday Trans. 1, 1975, 71, 1327-1334.

62 P. M. Holland and D. N. Rubingh, J. Phys. Chem., 1983, 87, 1984-1990.

63 J. A. Hammons, T. Muselle, J. Ustarroz, M. Tzedaki, M. Raes, A. Hubin and H. Terryn, J. Phys. Chem. C, 2013, 117, 14381-14389.

64 O. S. Hammond, K. J. Edler, D. T. Bowron and L. TorrenteMurciano, Nat. Commun., 2017, 8, 14150.

65 S. Kewalramani, H. Hlaing, B. M. Ocko, I. Kuzmenko and M. Fukuto, J. Phys. Lett., 2010, 1, 489-495.

66 E. K. Perttu and F. C. Szoka, Chem. Commun., 2011, 47, 12613-12615.

67 J.-P. Hansen and J. B. Hayter, Mol. Phys., 1982, 46, 651-656. 68 M. A. Gebbie, A. M. Smith, H. A. Dobbs, A. A. Lee, G. G. Warr, X. Banquy, M. Valtiner, M. W. Rutland, J. N. Israelachvili, S. Perkin and R. Atkin, Chem. Commun., 2017, 53, 1214-1224.

69 J. A. Hammons, J. Ustarroz, T. Muselle, A. A. J. Torriero, H. Terryn, K. Suthar and J. Ilavsky, J. Phys. Chem. C, 2016, 120, 1534-1545. 